\title{
Psychometric evaluation of an Italian custom 4-item Short Form of the PROMIS Anxiety item bank in immune-mediated inflammatory diseases: an item response theory analysis.
}

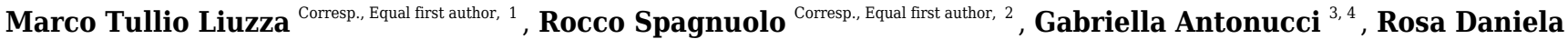 \\ Grembiale $^{5}$, Cristina Cosco ${ }^{2}$, Francesco Salvatore Iaquinta ${ }^{5}$, Vanessa Funari ${ }^{5}$, Stefano Dastoli ${ }^{5}$, Steven Nistico ${ }^{5}$, \\ Patrizia Doldo ${ }^{2}$ \\ 1 Department of Medical and Surgical Sciences, "Magna Graecia" University of Catanzaro, Catanzaro, Calabria, Italy \\ 2 Department of Clinical and Experimental Medicine, "Magna Graecia" University of Catanzaro, Catanzaro, Calabria, Italy \\ 3 IRCCS Santa Lucia Foundation, Rome, Lazio, Italy \\ 4 Department of Psychology, University of Roma "La Sapienza", Rome, Lazio, Italy \\ 5 Department of Health Sciences, "Magna Graecia" University of Catanzaro, Catanzaro, Calabria, Italy \\ Corresponding Authors: Marco Tullio Liuzza, Rocco Spagnuolo \\ Email address: liuzza@unicz.it, spagnuolo@unicz.it
}

Background: There has recently been growing interest in the roles of inflammation in contributing to the development of anxiety in people with Immune-mediated inflammatory diseases (IMID). Patient-reported outcome measures can facilitate the assessment of physical and psychological functioning. The National Institutes of Health (NIH)'s PatientReported Outcomes Measurement Information System (PROMIS ${ }^{\circledR}$ ) is a set of PatientReported Outcomes (PROs) that cover physical appearance, mental health, and social health. The PROMIS has been built through an Item Response Theory approach (IRT), a model-based measurement in which trait level estimates depend on both persons' responses and on the properties of the items that were administered. The aim of this study is to test the psychometric properties of an Italian custom 4-item Short Form of the PROMIS Anxiety item bank in a cohort of outpatients with IMIDs. Methods: We selected 4 items from the Italian standard Short Form Anxiety $8 a$ and administered them to consecutive outpatients affected by Inflammatory Bowel disease $(n=246)$, rheumatological $(n=100)$ and dermatological $(n=43)$ diseases, and healthy volunteers ( $n$ $=280$ ). Data was analyzed through an Item Response Theory (IRT) analysis in order to evaluate the psychometric properties of the Italian adaptation of the PROMIS anxiety short form.Results: Taken together, Confirmatory Factor Analysis and Exploratory Factor analysis suggest that the unidimensionality assumption of the instrument holds. The instrument has excellent reliability from a Classical Theory of Test (CTT) standpoint (Cronbach's $\alpha=.93$, McDonald's $\omega=.92$ ). The 2PL Graded Response Model (GRM) model provided showed a better goodness of fit as compared to the 1PL GRM model, and local 
independence assumption appears to be met overall. We did not find signs of Differential Item Functioning (DIF) for age and gender, but evidence for uniform (but not non-uniform) DIF was found in three out of four items for the patient vs. control group. Analysis of the test reliability curve suggested that the instrument is most reliable for higher levels of the latent trait of anxiety. The groups of patients exhibited higher levels of anxiety as compared to the control group ( $p s<.001$, Bonferroni-corrected). The groups of patients were not different between themselves ( $p=1$, Bonferroni-corrected). T-scores based on estimated latent trait and raw scores were highly correlated (Pearson's $r=.98$ ) and led to similar results.Discussion: The Italian custom 4-item short form from the PROMIS anxiety form 8a shows acceptable psychometric properties both from a CTT and an IRT standpoint. The Test Reliability Curve shows that this instrument is mostly informative for people with higher levels of anxiety, making it particularly suitable for clinical populations such as IMID patients. It is advised to compute T-scores based on the estimated latent trait in order to compare different groups. 
1 Psychometric evaluation of an Italian custom 4-item short form adaptation of the PROMIS

2 anxiety item bank in immune-mediated inflammatory diseases: an item response theory 3 analysis.

5 Marco Tullio Liuzza ${ }^{1 *}$ and Rocco Spagnuolo*2, Gabriella Antonucci ${ }^{3,4}$, Rosa Daniela

6 Grembiale $^{5}$, Cristina Cosco $^{1}$, Francesco Salvatore Iaquinta ${ }^{5}$, Vanessa Funari ${ }^{5}$, Stefano Dastoli ${ }^{5}$,

7 Steven Paul Nisticò ${ }^{5} \&$ Patrizia Doldo $^{1}$

8

9

1 "Magna Graecia” University of Catanzaro, Department of Medical and Surgical Sciences, Catanzaro, Italy

2 "Magna Graecia" University of Catanzaro, Department of Clinical and Experimental Medicine, Catanzaro,

12 Italy

3 IRCCS Santa Lucia Foundation, Rome, Italy

4."Department of Psychology, University of Roma "La Sapienza”, Rome, Italy

5 “Magna Graecia” University of Catanzaro, Department of Health Sciences, Catanzaro, Italy

* These authors contributed equally.

Corresponding Authors:

Marco Tullio Liuzza

Rocco Spagnuolo

Viale Europa (Loc. Germaneto), Catanzaro, CZ, 88100, Italy

Email addresses: 1iuzza@unicz.it and spagnuolo@unicz.it 


\section{Abstract}

32 Background: There has recently been growing interest in the roles of inflammation in contributing to the development of anxiety in people with Immune-mediated inflammatory diseases (IMID). Patient-reported outcome measures can facilitate the assessment of physical and psychological functioning. The National Institutes of Health (NIH)'s Patient-Reported Outcomes Measurement Information System (PROMIS ${ }^{\circledR}$ ) is a set of Patient-Reported Outcomes (PROs) that cover physical health, mental health, and social health. The PROMIS has been built through an Item Response Theory approach (IRT), a model-based measurement in which trait level estimates depend on both persons' responses and on the properties of the items that were administered. The aim of this study is to test the psychometric properties of an Italian custom 4item Short Form of the PROMIS Anxiety item bank in a cohort of outpatients with IMIDs.

Methods: We selected 4 items from the Italian standard Short Form Anxiety 8a and administered them to consecutive outpatients affected by Inflammatory Bowel disease $(\mathrm{n}=246)$, rheumatological $(n=100)$ and dermatological $(n=43)$ diseases, and healthy volunteers $(n=$ 280). Data was analyzed through an Item Response Theory (IRT) analysis in order to evaluate the psychometric properties of the Italian adaptation of the PROMIS anxiety short form.

Results: Taken together, Confirmatory Factor Analysis and Exploratory Factor analysis suggest that the unidimensionality assumption of the instrument holds. The instrument has excellent reliability from a Classical Theory of Test (CTT) standpoint (Cronbach's $\alpha=.93$, McDonald's $\omega$ $=.92)$. The 2PL Graded Response Model (GRM) model provided showed a better goodness of fit as compared to the 1PL GRM model, and local independence assumption appears to be met overall. We did not find signs of Differential Item Functioning (DIF) for age and gender, but evidence for uniform (but not non-uniform) DIF was found in three out of four items for the patient vs. control group. Analysis of the test reliability curve suggested that the instrument is most reliable for higher levels of the latent trait of anxiety. The groups of patients exhibited 
56 higher levels of anxiety as compared to the control group ( $p \mathrm{~s}<.001$, Bonferroni-corrected). The 57 groups of patients were not different between themselves ( $p=1$, Bonferroni-corrected). Factor 58 scores based on estimated latent trait through the EAP method and raw scores were highly 59 correlated (Pearson's $r=.98$ ) and led to similar results.

60 Discussion: The Italian custom 4-item short form from the PROMIS anxiety form 8a shows 61 acceptable psychometric properties both from a CTT and an IRT standpoint. The Test Reliability 62 Curve shows that this instrument is mostly informative for people with higher levels of anxiety, 63 making it particularly suitable for clinical populations such as IMID patients. It is advised to 64 compute T-scores based on the estimated latent trait in order to compare different groups.

65

66 68

69 70

71

Keywords: PROMIS; Item Response Theory; Graded Response Model; Anxiety; Rheumatoid arthritis; Psoriasis; Inflammatory Bowel Disease; Psychometrics; Quality of Life; Scale validation 


\section{Introduction}

The prevalence of anxiety is three times greater in patients with chronic diseases than in the general population (Abbott et al., 2015). In such patients, presence of these psychological disorders can be associated with an increase in mortality, in morbidity (Ellis et al., 2010; Nicholson et al., 2006), and, importantly, a deterioration in quality of life (Dickens et al., 2008; Dickens et al., 2004; Moussavi et al., 2007) with a consequent increase in the costs of healthcare. Disease and treatment factors are related to the determination of anxiety and depression, such as negative beliefs about the disease, the presence of pain, disability, and unpleasant side effects of treatment (Dickens et al., 2008; Harrison et al., 1994).

There has recently been growing interest in the interplay between inflammation and mood disorders. Potential mechanisms involved include direct effects of pro-inflammatory 111 cytokines monoamine levels, dysregulation of the hypothalamic-pituitary-adrenal (HPA) axis, 112 pathologic microglial cell activation, impaired neuroplasticity and structural and functional brain 113 changes (Rosenblat et al., 2014). Moreover, current evidence have shown that mood disorders 114 are expected from higher levels of inflammatory mediators such as IL-6 and TNF- $\alpha$ (Himmerich 115 et al., 2008; Howren et al., 2009; Schmidt et al., 2014) and have shown improved outcomes in 116 patients when anti-inflammatory agents such as Acetyl-salicylic acid (ASA), celecoxib, anti117 TNF-aagents, minocycline,curcumin and omega-3 fatty acid are used as an adjunct to 
118 conventional therapy. Inflammation contributes to the development of anxiety in people with

119 Immune-mediated inflammatory diseases (IMIDs), a group of ostensibly unrelated conditions

120 that share common inflammatory pathways encompasses about 80 diseases, including

121 Inflammatory bowel disease (IBD), Rheumatologic, and dermatological disorder (Kuek et al., 122 2007).

123 A recent systematic review and meta-analysis of randomized controlled trials regarding 124 the effects of TNF- $\alpha$ inhibitor therapy on depression and anxiety in people with chronic physical 125 illness, has shown that out of a total of 2540 patients affected by rheumatic and dermatological 126 diseases, treatment with anti-TNF-alpha induces a small reduction in depression $(-0.24 ; 95 \%$ CI $127-0.33$ to $-0.14 ; p<.001)$, and anxiety $(-0.17 ; 95 \% \mathrm{CI}-0.31$ to $-0.02 ; p=.02$, Abbott et al., 128 2015) Also in Inflammatory Bowel Diseases involving Crohn Disease (CD) and Ulcerative 129 Colitis (UC) psychological alterations have shown, due to dietary restrictions, long-term use of 130 medication to control the disease (Devlen et al., 2014), an immediate and lifelong psychological 131 impact on daily activities, such as absences from school or work, and difficulties with meeting 132 employment requirements. This leads to a reduction in Health-Related Quality of Life (HRQoL) 133 not only during the relapse phases but also during the periods of remission of the inflammatory 134 disease (Mancina et al., 2020).

A recent prospective cross-sectional study has shown that the prevalence of and factors associated with depression and anxiety in patients with rheumatoid arthritis (RA) are common.

137 Functional disability and marital status were significantly associated with increased risk, whereas 138 disease duration of 10 years or more and global health scores were significantly associated with decreased risk of developing anxiety (Katchmart et al., 2020).

140 In dermatological disorder, most of the evidence comes from psoriasis. Psychological 141 comorbidity, suboptimal coping, and low levels of well-being are associated with psoriasis, 142 significantly impacting on patients' lives (Esaa et al., 2020).

143 In recent years various patient outcome measures (PROMs) have been implemented: they 144 define the patient's experience with a disease and its treatment, including impressions, 145 perceptions, and attitudes, in addition, they are measures of the outcome of the disease 146 management reported directly by the patient or, alternatively, by the caregiver (Bojic et al., 147 2017). Furthermore, PROMs can facilitate the assessment of physical and psychological 148 functioning and identify suboptimal coping strategies. Over the years, several questionnaires and 
149 survey tools have been developed, including HRQoL tools, review of opinions and feedback 150 from patients with inflammatory bowel disease (IBD). Evaluation of PROMs in rheumatoid 151 arthritis (RA) was developed in the Outcome Measures in Rheumatology Clinical Trials 152 (OMERACT), leading to the definition of an international standard "core set"used in clinical 153 trials on RA (Cella et al., 2007; Wolfe et al., 1996).

154 No specific PRO measure is consistently used in the current management of psoriasis. A 155 recent systematic review (Duvetorp et al., 2019) revealed several limitations of the existing 156 measures. In 2019 a survey conducted on 22,050 adults affected by psoriasis and psoriatic 157 arthritis skin showed a negative impact on HRQOL, with $16 \%$ of patients reported anxiety and 158 depression disorder (El-Matary, 2014).

The National Institutes of Health (NIH)'s Patient-Reported Outcomes Measurement Information System (PROMIS ${ }^{\circledR}$, http://www.nihpromis.gov, Cella et al., 2007)) is a set of publicly available and standardized Patient-Reported Outcomes (PROs) that cover physical appearance, mental health, and social health. Two recent studies (Conley et al., 2018) on 5296 patients with IBD from the Crohn's and Colitis Foundation of America's Partners Cohort have used PROMIS to evaluate pain, fatigue, sleep disturbances, anxiety, and depression to identify symptom cluster membership among adults with IBD and examine associations between demographic and clinical factors. In 2015, a large prospective study (Bartlett et al., 2015), carried out in the US, provided preliminary evidence of reliability and construct validity of PROMIS to assess RA symptoms and impacts, and feasibility of use in clinical care. PROMIS instruments captured the experiences of RA patients across the broad continuum, especially at low disease activity levels. Finally, a retrospective multi-center study (Esaa et al., 2020) conducted on approximately 7000 patients with dermatological diseases showed that high $\mathrm{T}$ scores $>55$ measured by PROMIS were significantly correlated with severe disease activity, treatment failure, and uncontrolled 174 disease.

The flexibility and efficiency of the PROMIS relies on an Item Response Theory (IRT) approach (van der Linden, 2017). Indeed, IRT methods allow a deeper understanding of the items' relationships to the targeted domain. An IRT-based evaluation of items' psychometric

178 properties allows the investigators to create short-form scales or even computerized adaptive 179 tests for more targeted patient assessment (Revicki et al., 2009). 
IRT is "a model-based measurement in which trait level estimates depend on both

181 persons' responses and on the properties of the items that were administered (Embretson, 2013).

182 IRT models differ in terms of the number of parameters that model the relationship between the 183 latent trait and the (probability of) response.

184 One of the most straightforward ways to validate an instrument using an IRT approach is 185 to test whether data fit well with a Rasch model (Rasch, 1980), namely a model where the 186 probability of responding to an item (or, in polytomous items, to a category within each item) is 187 determined only by the difficulty of that item (and of that category), which is on the same scale 188 as the latent trait. Although the so-called one-parameter logistic models (1PL) within the IRT 189 approach are theoretically distinct from the Rasch model, they are mathematically equivalent, 190 and they both imply some desirable measure that matters in this context. Such a model implies 191 that all the items equally discriminate between respondents with low vs. high levels of ability 192 trait, and therefore the total sum score can be considered sufficient statistics of the latent trait 193 level.

194 On the other hand, in the two-parameter logistic model (2PL), an item discrimination 195 parameter is included in the measurement model. The 2PL model is more flexible and might be 196 useful when developing a new scale, for instance, by discarding the least discriminative items. 197 However, under this model, the items can weigh differently in determining the total score, and 198 therefore it does not retain all the desirable properties that characterize the Rasch model. 199 Comparing the fit of a 1PL model to a 2PL model serves as a way to test the hypothesis that the 200 data fits well enough to the Rasch model. If so, we can assume that the custom 4-items from the 201 Italian standard Short Form Anxiety 8a can provide a sufficient statistic for measuring anxiety 202 levels in the Italian context. On the other hand, the PROMIS was validated using a 2PL 203 approach, therefore a better fit for this model would be in accordance with the validation of this 204 instrument, although this would lead to a slightly different interpretation of the total score. 205

Another core feature that distinguishes this approach to measurement from the classical 207 test theory (Remmers, 1951; Spearman, 1907, 1913), is that the test reliability (or information, in 208 IRT parlance) differs between different levels of the latent trait. In fact, the relationship between information and standard error (SE) is defined by the formula $S E(\theta)=1 / \sqrt{I(\theta)}$, where $\theta$ is

210 estimated latent trait level, $\mathrm{SE}$ is the standard error of $\theta$, and $\mathrm{I}$ is information. Such a feature 
211 might be highly desirable when choosing items aimed at populations that should be either very

212 high or very low in certain latent traits.

213 Finally, another compelling reason for using an IRT approach is because the PROMIS

214 was developed using this approach (Cella et al., 2010). Indeed, through this approach it is

215 possible to use a small number of items without sacrificing the reliability of the instrument

216 (Embretson, 2013, Chapter 2). In this study, we used IRT analyses to test the psychometric

217 properties of the original anxiety items of the PROMIS

218

219 Materials \& Methods

220 Study Cohort

221 Between October 2018 and October 2019, 286 consecutive patients were enrolled in

222 Gastroenterology, Rheumatology and Dermatology Departments of the University Hospital

223 "Magna Graecia” University of Catanzaro. In addition, 280 healthy controls, carers of hospital

224 patients without cardiovascular, nor dysmetabolic disease were recruited. All patients were over

225 the age of 18 and had signed informed consent. At the time of enrollment, study participants

226 were invited by specialist nurses to complete questionnaires on anxiety through PROMIS, as

227 described below.

228 Demographic and anthropometric characteristics, smoking use, physical activity, marital status,

229 level of education, and job activity were recorded for the whole cohort of patients and healthy 230 controls.

231 Patients from each department with an established clinical, endoscopic, radiological, and 232 histological diagnosis underwent a full evaluation of disease characteristics: disease duration, 233 Erythrocyte Sedimentation Rate (ESR), Reactive C Protein (RCP) and previous surgery. Intake 234 of the drugs was collected in each patient. Assumption of not Steroidal Anti-Inflammatory 235 Drugs (NSAID), Mesalamine, Steroids, Disease-modifying antirheumatic drugs (DMARDs) 236 such as Methotrexate and azathioprine and biological therapy was recorded for each patient. 237 Harvey Bradshaw index (HBI; Harvey \& Bradshaw, 1980) for CD and Mayo Score (MS; 238 Rutgeerts et al., 2005) for UC, respectively have been considered for disease activity. Patients 239 were defined in remission when HBI $<5$ for $\mathrm{CD}$ or MS $<2$ for UC. Patients with 240 rheumatological diseases were divided into five groups: Axial Arthritis, Peripheral Arthritis, 241 Systemic Sclerosis, Systemic Lupus Erythematosus (SLE), and Vasculitis. Ankylosing 
242 Spondylitis Disease Activity Score (ASDAS; Lukas et al., 2009) index was used for axial 243 arthritis: ASDAS > 1.3 indicates active disease; Disease Activity Score -28 (DAS-28; Aletaha et 244 al., 2005) was used for peripheral arthritis: DAS $28>2.6$ indicates active disease; Medsger 245 Severity Index (Medsger et al., 2003) was used for Systemic Sclerosis and Selena-Systemic 246 Lupus Erythematosus Disease Activity Index (SELENA-SLEDAI; Petri, 2007) to assess the 247 degree of activity of SLE: Medsger Severity Index $>1$ and SELENA- SLEDAI $>5$ indicate 248 active disease. Birmingham Vasculitis Activity Score (Mukhtyar et al., 2009) to evaluate 249 vasculitis: score $>1$ indicates active disease.

250 Psoriasis Activity Score Index (PASI; Walsh et al., 2018) and Eczema Area Severity Index 251 (EASI, Simpson et al., 2016) has been used to calculate disease activity in psoriasis and atopic 252 dermatitis, respectively. In both cases, the disease is defined as active if the scores are $>0$.

253

254

Instruments

We selected 4 items from the Italian standard Short Form Anxiety 8a, a previously approved translated version. In this instrument, participants had to rate how often they experienced each of the symptoms (e.g., "I felt nervous") as occurring over the past seven days on a 5-points Likert response format ranging from 0 (Never) to $5=($ Always $)$.

Data analysis

261

262

\section{Unidimensionality}

263

To assess the unidimensionality assumption, we pursued a two-fold strategy.

Firstly, we tested whether the unidimensionality assumption for each sub-scale was met.

To do so, we pursued a two-fold strategy by conducting a confirmatory factor analysis (CFA) using the lavaan library (Rosseel, 2012) in R. Given the ordinal nature of the data, we used the Diagonally Weighted Least Squares (DWLS) robust estimator. We evaluated the model fit considering the Comparative Fit Index (CFI), Tucker-Lewis Index (TLI), Root-Mean-Square Error of Approximation (RMSEA), Standardized) Root Mean Square (SRMR), and Weighted Root Mean Square Residual (WRMR). We considered cut-off values as adequate if CFI and TLI were >.90, RMSEA less than .06 (Hu \& Bentler, 1999), and WRMR less than 1.0 (Muthén \& Muthén, 2010). 

polychoric correlations (extraction method = "minres") across the items in each subscale. Then,

275

276

277

278

279

280

281

282

283

284

285

286

287

288

289

290

291

292

293

294

295

296

297

298

299

300

301

302

we examined the ordered eigenvalues from the item correlation matrix (May, 1993). When the unidimensionality assumption is tenable for a subscale, the first eigenvalue should be considerably larger than the remaining eigenvalues. To this purpose, we used the fa.irt function provided by the psych library (Revelle, 2020) in $R$ (R Core Team, 2019).

We evaluated internal consistency through ordinal Cronbach's $\alpha$ computed from the polychoric correlation and through McDonald's $\omega$ computed from the unidimensional CFA model.

\section{IRT analysis}

Similarly to Fraley, Warren and Brennan (2000), we used a Graded Response Model (GRM, Samejima, 1968), an IRT model for polytomous categorical ordered responses (such as Likert-type responses). GRM is a so-called "indirect" item response theory (IRT) model that extends the 2PL for dichotomous item responses. As described in Fraley et al. (2000), within the GRM framework, an item response scale is conceptualized as a series of $k-1$ response dichotomies, where $k$ represents the number of response options for a given item. Samejima's model considers the probability of endorsing each response option category, or higher as a function of a latent trait $\left[P_{x i}(\theta)\right]$. The response option difficulty represents the point on the latent trait continuum where there is a 50\% chance of endorsing the or higher response option. In this sense, the response option difficulty represents a between-option "threshold" parameter. For each item, the number of threshold values equals the number of response dichotomies $(k-1)$. In the 2PL GRM, each item has a single discrimination $(\alpha)$ value for all response options. However, the discrimination parameter can be constrained to be equal across items, so to reach some of the desirable mathematical properties of the Rasch model.

GRM models were fitted and analyzed through the mirt package (Chalmers, 2012), which fits the models using Marginal Maximum Likelihood Estimation (MMLE). We fit two models, one with the discrimination constrained to be equal, and the other one without that constraint, namely allowing different slopes for each item. We compared the two models using a Likelihood Ratio test, but we also looked at the Bayesian information criterion (Schwarz, 1978), which favors parsimonious models over complex ones.

Peer] reviewing PDF | (2020:05:49173:2:1:NEW 19 Jun 2021) 
The goodness of fit was tested through the M2* statistics proposed by Cai and Hansen 304 (2013), and by looking at absolute (Root Mean Square of Error Approximation, RMSEA; 305 Standardized Root Mean Square Residual, SRMSR) and comparative (Comparative Fit Index, CFI; Tucker-Lewis Index, TLI) indices. Absolute indices should be the closest to zero as possible, but a good fit is achieved with values $<.05$, whereas comparative fit indices should approach the value of 1 , and they are considered good if $>0.95$ (Hu \& Bentler, 1999).

We tested the assumption of local independence by using the Q3 statistics (Yen, 1984), which checks whether two items are correlated after controlling for the latent trait level. These correlations are deemed as problematic if their absolute value exceeds .2 (Yen, 1993).

We assessed the Differential item functioning (DIF) using the ordinal logistic regression method (Zumbo, 1999). This method is quite straightforward, as it tests for whether adding the effect of the group, and its interaction with theta scores significantly changes the explained variance of a model in which theta scores were entered first. Through this method, a significant effect of the group is a sign of uniform DIF, whereas a significant interaction between theta scores and the group is a sign of non-uniform DIF. On top of that, we assessed also the effect size of the DIF by checking the McFadden pseudo-R ${ }^{2}$ (Menard, 2000) change. To this purpose DIF analyses were conducted using the lordif (Choi et al., 2016) package in R, as kindly suggested by a Reviewer.

321 We assessed DIF as a function of demographic variables such as gender and age (65 years vs. 65 years or older (Pilkonis et al., 2011), and for the groups of patients (patients with inflammatory bowel disease, IBD; dermatological patients, DER, and rheumatological patients, RHE) vs. the

324 Control group (CTR).

\section{Group differences}

We computed the factor scores (estimated latent trait) levels through the expected a posteriori method (EAP) from the 2PL model. To test whether the groups (CTR, IBD, DER and RHE) differed in anxiety levels, we conducted an ANOVA on the factor scores, controlling for gender and age. We followed-up the omnibus analysis through a set of six post-hoc pairwise comparisons (Bonferroni-corrected). Furthermore, we computed the T-scores on the basis of the parameters estimated in the PROMIS normative data, computed through the Health Measures

333 Scoring Service (https://www.assessmentcenter.net/ac scoringservice) and conducted the same 
334 analyses as for the factor scores. Briefly, we inserted our short form by selecting from the

335 PROMIS Item Bank V1.0 -Anxiety, we selected the calibration sample PROMIS Wave 1 and 336 upload our dataset using the template provided.

337

338

339

Ethics

340

The Calabria Region regional ethics committee approved this study on 15 March 2018

341 (protocol number 69). All patients participated in this study in confirmation with the principles outlined in the Declaration of Helsinki. We obtained informed written consent from each 343 participant.

Data availability

Data and R scripts are available on the Open Science Framework platform at this link: https://osf.io/92ykx/

348

\section{Results}

Descriptive statistics

First, we identified one observation in which an anomalous response was recorded (i.e., responses above five on a 5 points Likert-type response format), and coded it as missing data.

We show demographic and anthropometric characteristics of patients stratified by disease and controls in Table 1. Briefly, out of a total of 566, 286 were patients, and 280 were the healthy controls. Two hundred ninety-one (51\%) were male, with a median age of $49 \pm 16$ and a median $\mathrm{BMI}=25 \pm 4 \mathrm{~kg} / \mathrm{m} 2$. One hundred ninety-eight (35\%) carried out physical activity. Only 100 $(18 \%)$ was a graduate, $211(38 \%)$ had a job, and $179(32 \%)$ were married.

We show disease characteristics in Table 2. One hundred thirty-six (47\%) were suffering from IBD, 100 (35\%) were suffering from rheumatic diseases, and 42 (15\%) had dermatological diseases. The mean duration of disease was $10 \pm 8$ years, and approximately $29 \%$ (80) had active

361 disease. One hundred eighty-three (64\%) received mesalazine, 53 (19\%) received steroids, and, 362 finally, $95(23 \%)$ received biologic therapy.

A visual check on the response distributions in the four items shows that most of them display a positively skewed distribution because most of the respondents refer to have never 
365 experienced anxiety-related symptoms (see Figure 1). Such a pattern was expected and, in fact,

366

367

368

369

370

371

372

373

374

375

376

377

378

379

380

381

382

383

384

385

386

387

388

389

390

391

392

393

parallels the findings from the original item bank construction study.

\section{Dimensionality}

The confirmatory factor analysis on the unidimensional model showed a good fit in terms of CFI and TLI (both equal to 1), SRMR (.007) RMSEA (0), and WRMR (0.24).

Results from the EFA on the polychoric correlations show a dominant first dimension (see Figure 2), and a one-factor solution is well justified by both a scree test approach and a mineigen approach (eigenvalues $>1$ ). Overall, taking the EFA and the CFA analyses together, the unidimensional solution seems well-justified. Therefore, we decided to pursue the following IRT analyses assuming unidimensionality (see Figure 2). The items showed excellent reliability both in terms of polychoric Cronbach's $\alpha$ computed from the polychoric (.93) correlation and through McDonald's $\omega$ computed from the unidimensional CFA model (.92).

\section{Graded response models}

The Likelihood ratio test showed a significant improvement with the unconstrained model (2PL), even in terms of BIC (see Table 3). Moreover, we conducted a M2* test (Cai and Hansen, 2013, C2 variant from Cai and Monro, 2014) to test the goodness of fit of our model, which did not reject the 2PL model (4.57, $\mathrm{df}=2, p=.1$ ), all the other fit indices were good $(\mathrm{RMSEA}=.05, \mathrm{SRMSR}=.02, \mathrm{CFI}=1, \mathrm{TLI}=1)$. The reason why the $2 \mathrm{PL}$ model fits the data better, is that there is a noticeable variability in the item discrimination parameters, with the first item ("I felt uneasy") having the lowest discrimination (alfa = 2.58) and the third item ("I felt anxious") having the highest (alfa $=5.95)$. An inspection of the factor loadings computed from the slope parameters showed that all the items had excellent loadings ( $>.7)$. A visual inspection of the Item Response Category Characteristic Curves shows that the assumption of monotonicity was met (see Figure 3).

The single Item Information Curves and the overall test reliability curve (Figure 4) computed from the test information curve showed that the scale is most reliable for values of the latent trait that are just below zero up to two logits, meaning that the scale is most reliable for 
394 higher levels of anxiety.

395 The inspection of the Q3 residuals showed that some pairs of items have an absolute

396 value higher than .2. This result suggests that local independence conditional on the latent trait

397 seems to be violated, especially for some items. However, most of these correlations were

398 negative, and this might be due to an artifact because the instrument is short (De Ayala, 2008).

399 When looking at the positive Q3 values, we found that none of the items were problematic.

400

401

\section{Differential item functioning}

402 In this analysis, we set the alpha value as $.05 / 4=.0125$ to correct for the Family-Wise error rate.

403 Gender We found no evidence for either uniform or non-uniform DIF ( $\left.p \mathrm{~s}^{-} \geq .0125\right)$

404 Age. We found no evidence for either uniform or non-uniform DIF ( $p \mathrm{~s} \geq .07$ ).

405 Groups of patients vs. Control group. We found evidence $(p<.0125)$ for a uniform and non406 uniform DIF in for all the items. However, when looking at the effect size for such DIF effects, 407 they were all small (MacFadden $\Delta R s^{2}<.13$ ).

Group differences in anxiety levels.

After computing the factor scores (estimated latent traits), we ascertained that the factor scores and the total sum scores displayed a very high correlation coefficient (Pearson's $r=.99$ ).

412 However, it is worth noting that, because the data do not conform to a Rasch model, the same 413 raw scores could reflect slightly different levels of latent traits and, therefore, of factor scores.

414 We found a significant effect of the group $(F(3,561)=19.3, p<.001)$ on factor scores.

415 The post-hoc comparison showed that the Control group displayed significantly lower 416 levels of anxiety levels ( $p \mathrm{~s},<.001$, Bonferroni-corrected) compared to all the other patent 417 groups. On the other hand, the patient groups did not significantly differ each from the other $p \mathrm{~s}=$ 418 1, Bonferroni-corrected). Running the same analyses on the raw scores led to the same results.

419 We conducted the same analyses on the T-scores, which were nearly perfectly correlated with 420 the factor scores computed locally $(r=.99)$. We found a significant effect of the group $(F(3,561)$ $421=18.57, p<.001$ ) on $\mathrm{T}$ - scores (see Figure 5).

422 The post-hoc comparison showed that the Control group displayed significantly lower levels of 423 anxiety levels ( $p \mathrm{~s}=<.001$, Bonferroni-corrected) compared to all the other patent groups. On the 424 other hand, the patient groups did not significantly differ each from the other $p \mathrm{~s}=1$, Bonferroni- 
425 corrected).

426

427

428

429

430

431

432

433

434

435

436

437

438

439

440

441

442

443

444

445

446

447

448

449

450

451

452

453

454

455

\section{Discussion}

In agreement with the Promis Health Organization, we tested for the first time a PROMIS anxiety custom 4-item short form in Italian adapted from the PROMIS Anxiety short form 8a. The use of patients reported outcomes to identify anxiety has numerous advantages in the context of chronic inflammatory diseases such as IBD, rheumatic and dermatological diseases. Measuring health outcomes is of pivotal importance in clinical practice. Quantifying in a standardized way is less time consuming and does not require trained personnel. The PROMIS database consists of a large items bank that has been extensively validated using an IRT approach (Cella et al., 2010) with the aim of goal creating and evaluating a set of publicly available, flexible, and efficient measurements of important symptoms and patient function that could be easily be used by the clinical research community.

In this study, we selected 4 items from the Italian standard Short Form Anxiety 8a and administered them an Italian sample $(\mathrm{n}=568,271 \mathrm{~F})$ sample that included Dermatological, Rheumatological patients, and IBD patients, using an Item Response Theory approach. Such a sample size is above the minimum recommended $(n=500)$ for fitting 2PL models (De Ayala, 2008).

First, we confirmed the unidimensionality of the measurement, using a CFA for categorical ordered variables in conjunction with an EFA on the polychoric correlation. This finding implies that these four items should reflect a specific construct, and this a precondition for evaluating the reliability using a unique estimate and for testing the psychometric properties of the items using an IRT approach.

Secondly, we conducted an IRT analysis for polytomous items using a modified version of Samejima's GRM. However, in a first step, we constrained the item discrimination to be equal in all the items. In the second step, we allowed for a free estimation of the discrimination. We found that the second improved the goodness of fit, the M2* test confirmed that a $2 \mathrm{PL}$ model could not be rejected. Such a finding suggests that our data are not compatible with a Rasch model, and therefore the unweighted sum score cannot be considered a sufficient statistic of participants' latent traits. Users are recommended to compute factor scores, instead. This is line 
456 with what was found in the development of the PROMIS, in which the developers explicitly 457 preferred the greater flexibility provided by a $2 \mathrm{PL}$ model as compared to a 1PL model. 458 Moreover, we found that, in practice, factor scores (estimated latent trait levels) and raw scores 459 were almost perfectly correlated.

460

Thirdly, we assessed the reliability using both Cronbach' $\alpha$ and McDonald's $\omega$, which both

462

463

464

465

466

467

468

469

470

471

472

473

474

475

476

477

478

479

480

481

482

483

484

485

486

showed excellent reliability in terms of internal consistency from a CTT standpoint. Moreover, our analysis of the test information showed that the test is most informative for higher levels of the latent trait. Overall, our results showed that this custom Italian 4-items short form not only reliability estimates anxiety levels but performs better with people who are higher in anxiety. Therefore, the test appears to be especially suited for testing clinical populations such as IBD, Dermatological, and Rheumatism patients.

Fourthly, the inspection of the assumption of local independence further corroborated the validity of this short measure .

Finally, the lack of DIF as a function of gender and age suggests that this shorter measure raw scores could be used to compare these groups. On the other hand, patients and controls exhibited uniform DIF in all the items, although the magnitude of this effect was small.

Overall, these results suggest that this short custom four-item short form in Italian could easily be used.

Coherently with previous data (Whitehouse et al., 2019) that showed that IMIDs share an increased prevalence of mood disorders such as anxiety and depression, when we compared the factor scores of the Control group with the other patients' groups, we found significantly lower levels of anxiety in the Control group, as compared to the patients' groups. However, patients affected by different disorders (either Dermatological, Rheumatological or Gastrointestinal) did not differ from each other. A possible limitation is that in this sample the patients' groups were oversampled in comparison to the control group. One of the advantages of the IRT approach is that item parameters estimation is independent from population assumptions, and that a representative sample is not necessary during the calibration (Embretson \& Reise, 2000; De Mars, 2010). However, it has been shown that, depending on different scenarios, oversampling of patients' population can bias parameter estimation in cases in which items show quasi-trait characteristics, namely when they are poorly informative in the low end of the distribution

PeerJ reviewing PDF | (2020:05:49173:2:1:NEW 19 Jun 2021) 
487 (Smith, 2016). Nevertheless, it is worth noting that, although this may represent a problem when 488 the sample is used for building norms, that was not the goal of the present study. Furthermore, 489 the latent traits (factor scores) estimated in our sample nearly perfectly correlated ( $r=.99)$ with 490 the T-scores computed using the parameters from the US normative sample. Finally, the DIF 491 analysis showed a less than moderate bias in the items' parameter estimations. Overall, 492 considering the purpose of the study, and taking empirical and pragmatical consideration into 493 account, the oversampling of the population of the patients should not threaten the validity of our 494 conclusions.

495

496

497

\section{Conclusions}

498

499

500

501

502

503

504

505

506

507

508

509

510

511

512

513

514

515

516

517

In the present study, we selected 4 items from the Italian standard Short Form Anxiety 8a and administered them to an Italian sample that included both healthy controls and IMIDs patients. We thus validated the anxiety custom 4-item PROMIS short form using an IRT approach. The results confirm that these items are well suited for measuring anxiety in clinical populations. Moreover, the use of a 4 items custom form could prove useful for the brevity and speed of execution in high flow clinics such as those of IMIDs. Further studies are needed to confirm that a shorter items version could be utilized.

\section{Acknowledgements}

The authors thank Ms. Sabrina Ligarò for data collection and data entry. We also wish to thank Roberto Giorgini for his help in the preparation of the tables.

\section{References}

Abbott, R., Whear, R., Nikolaou, V., Bethel, A., Coon, J. T., Stein, K., \& Dickens, C. (2015). Tumour necrosis factor- $\alpha$ inhibitor therapy in chronic physical illness: A systematic review 
518

519

520

521

522

523

524

525

526

527

528

529

530

531

532

533

534

535

536

537

538

539

540

541

542

543

544

545

546

and meta-analysis of the effect on depression and anxiety. In Journal of Psychosomatic Research. https://doi.org/10.1016/j.jpsychores.2015.04.008

Aletaha, D., Ward, M. M., Machold, K. P., Nell, V. P. K., Stamm, T., \& Smolen, J. S. (2005). Remission and active disease in rheumatoid arthritis: Defining criteria for disease activity states. Arthritis and Rheumatism. https://doi.org/10.1002/art.21235

Bartlett, S. J., Orbai, A. M., Duncan, T., DeLeon, E., Ruffing, V., Clegg-Smith, K., \& Bingham, C. O. (2015). Reliability and validity of selected PROMIS measures in people with rheumatoid arthritis. PLOS ONE. https://doi.org/10.1371/journal.pone.0138543

Bojic, D., Bodger, K., \& Travis, S. (2017). Patient reported outcome measures (PROMs) in inflammatory bowel disease: New data. Journal of Crohn's and Colitis. https://doi.org/10.1093/ecco-jcc/jjw187

Cai, L. \& Hansen, M. (2013). Limited-information goodness-of-fit testing of hierarchical

item factor models.ÂA British Journal of Mathematical and Statistical

Psychology,

$66,245-276$.

Cai, L. \& Monroe, S. (2014). A New Statistic for Evaluating Item Response Theory Models for Ordinal Data (CRESST Report 839). Los Angeles, CA: University of California, National Center for Research on Evaluation, Standards, and Student Testing (CRESST).

Cella, D., Riley, W., Stone, A., Rothrock, N., Reeve, B., Yount, S., Amtmann, D., Bode, R., Buysse, D., Choi, S., Cook, K., Devellis, R., Dewalt, D., Fries, J. F., Gershon, R., Hahn, E. A., Lai, J. S., Pilkonis, P., Revicki, D., ... Hays, R. (2010). The patient-reported outcomes measurement information system (PROMIS) developed and tested its first wave of adult self-reported health outcome item banks: 2005-2008. Journal of Clinical Epidemiology. https://doi.org/10.1016/j.jclinepi.2010.04.011 
548 Cella, D., Yount, S., Rothrock, N., Gershon, R., Cook, K., Reeve, B., Ader, D., Fries, J. F., 549 Bruce, B., \& Rose, M. (2007). The Patient-Reported Outcomes Measurement Information System (PROMIS): Progress of an NIH roadmap cooperative group during its first two

552

Chalmers, R. P. (2012). Mirt: A multidimensional item response theory package for the R environment. Journal of Statistical Software. https://doi.org/10.18637/jss.v048.i06

555

556

557

558 years. Medical Care. https://doi.org/10.1097/01.mlr.0000258615.42478.55

\section{9}

560

561

562

Conley, S., Jeon, S., Proctor, D. D., Sandler, R. S., \& Redeker, N. S. (2018). Longitudinal Changes in Symptom Cluster Membership in Inflammatory Bowel Disease. Journal of 563

De Ayala, R. J. (2008). The theory and practice of item response theory. Methodology in the 565 Social Sciences.

566

Devlen, J., Beusterien, K., Yen, L., Ahmed, A., Cheifetz, A. S., \& Moss, A. C. (2014). The 568 burden of inflammatory bowel disease: A patient-reported qualitative analysis and development of a conceptual model. Inflammatory Bowel Diseases.

571

Dickens, C. M., Percival, C., McGowan, L., Douglas, J., Tomenson, B., Cotter, L., Heagerty, A., 573 \& Creed, F. H. (2004). The risk factors for depression in first myocardial infarction patients. 575 https://doi.org/10.1097/01.MIB.0000440983.86659.81 Psychological Medicine. https://doi.org/10.1017/S0033291704001965

Dickens, C., McGowan, L., Percival, C., Tomenson, B., Cotter, L., Heagerty, A., \& Creed, F. (2008). Negative illness perceptions are associated with new-onset depression following 
578

579

580

581

582

583

584

585

586

587

588

589

590

591

592

593

594

595

596

597

598

599

600

601

602

603

604

605

606

607

608 myocardial

infarction.

General

Hospital

Psychiatry.

Duvetorp, A., Østergaard, M., Skov, L., Seifert, O., Tveit, K. S., Danielsen, K., \& Iversen, L. (2019). Quality of life and contact with healthcare systems among patients with psoriasis and psoriatic arthritis: results from the NORdic PAtient survey of Psoriasis and Psoriatic arthritis (NORPAPP). Archives of Dermatological Research. https://doi.org/10.1007/s00403-019-01906-z

El-Matary, W. (2014). Patient-reported outcome measures in inflammatory bowel disease. In Canadian Journal of Gastroenterology and Hepatology. https://doi.org/10.1155/2014/792386

Ellis, C., Zhao, Y., \& Egede, L. E. (2010). Depression and increased risk of death in adults with stroke. Journal of Psychosomatic Research. https://doi.org/10.1016/j.jpsychores.2009.11.006

Embretson, Susan E., R. S. P. (n.d.). Item response theory.Psychology Press.

Esaa, F., Prezzano, J., Pentland, A., \& Ryan Wolf, J. (2020). The utility of PROMIS domain measures in dermatologic care. Archives of Dermatological Research. https://doi.org/10.1007/s00403-020-02074-1

Flora, D. B. (2017). Statistical methods for the social and behavioural sciences: A model-based approach. SAGE.

Harrison, J., \& Maguire, P. (1994). Predictors of psychiatric morbidity in cancer patients. In British Journal of Psychiatry. https://doi.org/10.1192/bjp.166.4.538a

Harvey, R. F., \& Bradshaw, J. M. (1980). A Simple Index of Crohn's-Disease Activity. The Lancet. https://doi.org/10.1016/S0140-6736(80)92767-1 
610 Himmerich, H., Fulda, S., Linseisen, J., Seiler, H., Wolfram, G., Himmerich, S., Gedrich, K., 611 Kloiber, S., Lucae, S., Ising, M., Uhr, M., Holsboer, F., \& Pollmächer, T. (2008).

612 Depression, comorbidities and the TNF- $\alpha$ system. European Psychiatry. 613 https://doi.org/10.1016/j

614

615 Howren, M. B., Lamkin, D. M., \& Suls, J. (2009). Associations of depression with c-reactive 616 protein, IL-1, and IL-6: A meta-analysis. Psychosomatic Medicine. 617 https://doi.org/10.1097/PSY.0b013e3181907c1b

618

619

Hu, L. T., \& Bentler, P. M. (1999). Cutoff criteria for fit indexes in covariance structure analysis: 620 Conventional criteria versus new alternatives. Structural Equation Modeling. 621 https://doi.org/10.1080/10705519909540118

622

623

Katchmart, W., Narongoreknawin, P., Chanapai, W., Thaweeratthakul, P., \& Srisomnuek, A. 624

625 (2020). Prevalence of and Factors Associated With Depression and Anxiety in Patients With Rheumatoid Arthritis: A Multicenter Prospective Cross-Sectional Study. International 627 Journal of Rheumatic Diseases, 23(3), 302-308

Kuek, A., Hazleman, B. L., \& Östör, A. J. K. (2007). Immune-mediated inflammatory diseases (IMIDs) and biologic therapy: A medical revolution. In Postgraduate Medical Journal. 631

Lukas, C., Landewé, R., Sieper, J., Dougados, M., Davis, J., Braun, J., Van Der Linden, S., \& 633 https://doi.org/10.1136/pgmj.2006.052688

636

Mancina, R. M., Pagnotta, R., Pagliuso, C., Albi, V., Bruno, D., Garieri, P., Doldo, P., \& 638 Spagnuolo, R. (2020). Gastrointestinal symptoms of and psychosocial changes in inflammatory bowel disease: A nursing-led cross-sectional study of patients in clinical 
642 May, K. (1993). Book Review : Fundamentals of Item Response Theory Ronald K. Hambleton, 643 H. Swaminathan, and H. Jane Rogers Newbury Park CA: Sage, 1991, 174 pp. Applied 644 Psychological Measurement. https://doi.org/10.1177/014662169301700309

645

Medsger, T. A., Bombardieri, S., Czirjak, L., Scorza, R., Della Rossa, A., \& Bencivelli, W. 649

Moussavi, S., Chatterji, S., Verdes, E., Tandon, A., Patel, V., \& Ustun, B. (2007). Depression, 651

652

653

654 Mukhtyar, C., Lee, R., Brown, D., Carruthers, D., Dasgupta, B., Dubey, S., Flossmann, O., Hall, 655 (2003). Assessment of disease severity and prognosis. In Clinical and Experimental Rheumatology.

chronic diseases, and decrements in health: results from the World Health Surveys. Lancet. https://doi.org/10.1016/S0140-6736(07)61415-9 C., Hollywood, J., Jayne, D., Jones, R., Lanyon, P., Muir, A., Scott, D., Young, L., \& 659

Muthén, L., \& Muthén, B. (2010). Mplus 3.0 user’s guide. Los Angeles: Author.

661

662

Nicholson, A., Kuper, H., \& Hemingway, H. (2006). Depression as an aetiologic and prognostic 663 factor in coronary heart disease: A meta-analysis of 6362 events among 146538 664 participants in 54 observational studies. European Heart Journal. https://doi.org/10.1093/eurheartj/ehl338

666

Petri, M. (2007). Disease activity assessment in SLE: Do we have the right instruments? Annals 668 of the Rheumatic Diseases. https://doi.org/10.1136/ard.2007.078477

669 
671

672

673

674

675

676

677

678

679

680

681

682

683

684

685

686

687

688

689

690

691

692

693

694

695

696

697

698

699

700

701

banks for measuring emotional distress from the patient-reported outcomes measurement information system (PROMIS $\left.{ }^{\circledR}\right)$ : Depression, anxiety, and anger. Assessment. https://doi.org/10.1177/1073191111411667

R Core Team (2019). (2019). R: A language and environment for statistical computing. Accessed 1st April 2019.

Rasch, G. (1980). Probabilistic models for some intelligence and attainment tests (Exp. ed.). Chicago: University of Chicago Press. (Original work published 1960, Copenhagen: Danish Institute for Educational Research)

Remmers, H. H. (1951). Theory of Mental Tests. Harold Gulliksen. New York: Wiley; London: $\begin{array}{llllll}\text { Chapman } \quad \& \quad \text { Hall, } & 1950 . & 486 & \text { pp. } & \$ 6.00 . & \text { Science. }\end{array}$ https://doi.org/10.1126/science.113.2943.612

Revelle, W. (2020). Psych: Procedures for Personality, Psychometric, and Psychological Research. In January.

Revicki, D. A., Chen, W. H., Harnam, N., Cook, K. F., Amtmann, D., Callahan, L. F., Jensen, M. P., \& Keefe, F. J. (2009). Development and psychometric analysis of the PROMIS pain

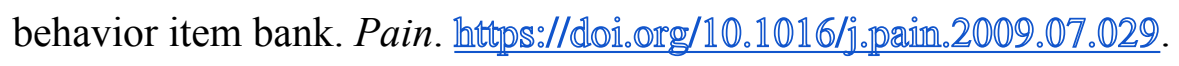

Rosenblat JD, Cha DS, Mansur RB, McIntyre RS. (2014) Inflamed moods: a review of the interactions between inflammation and mood disorders. Prog Neuropsychopharmacol Biol Psychiatry. 4;53:23-34. doi: 10.1016/j.pnpbp.2014.01.013.

Rosseel, Y. (2012). Lavaan: An R package for structural equation modeling. Journal of Statistical Software. https://doi.org/10.18637/jss.v048.i02

Rutgeerts, P., Sandborn, W. J., Feagan, B. G., Reinisch, W., Olson, A., Johanns, J., Travers, S., 
702

703

704

705

706

707

708

709

710

711

712

713

714

715

716

717

718

719

720

721

722

723

724

725

726

727

728

729

730

731

732

Rachmilewitz, D., Hanauer, S. B., Lichtenstein, G. R., De Villiers, W. J. S., Present, D., Sands, B. E., \& Colombel, J. F. (2005). Infliximab for induction and maintenance therapy for ulcerative colitis. New England Journal of Medicine. https://doi.org/10.1056/NEJMoa050516

Samejima, F. (1968). ESTIMATION OF LATENT ABILITY USING A RESPONSE PATTERN OF GRADED SCORES1. ETS Research Bulletin Series. https://doi.org/10.1002/j.2333-8504.1968.tb00153.x

Schmidt, F. M., Kirkby, K. C., \& Himmerich, H. (2014). The TNF-alpha inhibitor etanercept as monotherapy in treatment-resistant depression - Report of two cases. Psychiatria Danubina.

Schwarz, G. (1978). Estimating the Dimension of a Model. The Annals of Statistics. https://oi.org/10.1214/aos/1176344136

Simpson, E. L., Bieber, T., Guttman-Yassky, E., Beck, L. A., Blauvelt, A., Cork, M. J., Silverberg, J. I., Deleuran, M., Kataoka, Y., Lacour, J. P., Kingo, K., Worm, M., Poulin, Y., Wollenberg, A., Soo, Y., Graham, N. M. H., Pirozzi, G., Akinlade, B., Staudinger, H., ... Ardeleanu, M. (2016). Two Phase 3 Trials of dupilumab versus placebo in atopic dermatitis. New England Journal of Medicine. https://doi.org/10.1056/NEJMoa1610020

Spearman, C. (1907). Demonstration of Formulae for True Measurement of Correlation. The American Journal of Psychology. https://doi.org/10.2307/1412408

Spearman, C. (1913). Correlations Of Sums Or Differences. British Journal of Psychology, 1904-1920. https://doi.org/10.1111/j.2044-8295.1913.tb00072.x

Thissen, D., Steinberg, L., \& Wainer, H. (1993). Detection of differential item functioning using the parameters of item response models. In Differential Item Functioning

van der Linden, W. J. (2017). Handbook of Item Response Theory (C. and Hall/CRC (ed.) 
733

734 Walsh, J. A., Jones, H., Mallbris, L., Callis Duffin, K., Krueger, G. G., Clegg, D. O., \& Szumski, 735 A. (2018). The Physician Global Assessment and Body Surface Area composite tool is a simple alternative to the Psoriasis Area and Severity Index for assessment of psoriasis: post hoc analysis from PRISTINE and PRESTA. Psoriasis: Targets and Therapy.

Whitehouse, C. E., Fisk, J. D., Bernstein, C. N., Berrigan, L. I., Bolton, J. M., Graff, L. A., 741 https://doi.org/10.2147/ptt.s169333 Hitchon, C. A., Marriott, J. J., Peschken, C. A., Sareen, J., Walker, J. R., Stewart, S. H.,

Wolfe, F., Hawley, D. J., \& Wilson, K. (1996). The prevalence and meaning of fatigue in rheumatic disease. Journal of Rheumatology

Yen, W. M. (1984). Effects of Local Item Dependence on the Fit and Equating Performance of 752 the Three-Parameter Logistic Model. Applied Psychological Measurement. https://doi.org/10.1177/014662168400800201

754 dependence. Journal of educational measurement, 30(3), 187-213 
764

765

766

767

768

769

770

771

772

773

774

775

776

777

Peer] reviewing PDF | (2020:05:49173:2:1:NEW 19 Jun 2021) 


\section{Table $\mathbf{1}$ (on next page)}

Table 1. Characteristics of patients stratified by study group.

Continuous variables are shown as mean \pm standard deviation, categorical variables are presented as number and proportion. Abbreviations: BMI: Body Mass Index. CRT: Control, IBD: Inflammatory Bowel Disease, Rheuma: Rheumatological Disorders, Derma:

Dermatological Disorders 
Table 1. Characteristics of patients stratified by study group.

\begin{tabular}{|c|c|c|c|c|}
\hline & CTR & IBD & RHE & DER \\
\hline$\underline{\mathbf{N}}$ & 280 & 136 & 100 & 42 \\
\hline \multicolumn{5}{|c|}{ Demographic and Anthropometric } \\
\hline Male gender n (\%) & $162(58)$ & $85(63)$ & $25(25)$ & $19(47)$ \\
\hline $\mathrm{BMI}\left(\mathrm{Kg} / \mathrm{m}^{2}\right)$ & $25 \pm 3$ & $25 \pm 4$ & $26 \pm 4$ & $24 \pm 3$ \\
\hline Age (years) & $47 \pm 18$ & $49 \pm 13$ & $57 \pm 12$ & $51 \pm 15$ \\
\hline Smoke Y n (\%) & $124(44)$ & $27(20)$ & 49 (49) & $10(25)$ \\
\hline Phisical Activity Y n ( \%) & $104(37)$ & $53(39)$ & $26(26)$ & $15(38)$ \\
\hline \multicolumn{5}{|l|}{ Education level n (\%) } \\
\hline Diploma & $177(63)$ & $93(69)$ & 49 (49) & $20(50)$ \\
\hline Degree & $61(22)$ & $16(12)$ & $18(18)$ & $5(12)$ \\
\hline \multicolumn{5}{|l|}{ Marital status n (\%) } \\
\hline Single & $117(42)$ & $29(22)$ & $23(22)$ & $11(28)$ \\
\hline Married & $146(52)$ & $107(78)$ & $78(78)$ & $27(67)$ \\
\hline \multicolumn{5}{|l|}{ Occupation n (\%) } \\
\hline Employee & $100(35)$ & $72(53)$ & $27(27)$ & $12(28)$ \\
\hline
\end{tabular}

Continuous variables are shown as mean \pm standard deviation, categorical variables are presented as number and proportion. Abbreviations: BMI: Body Mass Index. CRT: Control IBD: Inflammatory Bowel Disease, RHE: Rheumatological Disorders, DER:Dermatological Disorders 


\section{Table 2 (on next page)}

Table 2. Disease characteristics

Continuous variables are shown as mean \pm standard deviation, categorical variables are presented as number and proportion. Abbreviations: HBI: Harvey Bradshaw Index; MS: Mayo Score; ASDAS: Ankylosing Spondylitis Disease Activity Score; DAS-28: Disease Activity Score -28 ;MSI: Medsger Severity Index; SELENA-SLEDAI: Selena-Systemic Lupus Erythematosus Disease Activity Index; BVAS: Birmingham Vasculitis Activity Score; PASI: Psoriasis Activity Score Index; EASI: Eczema Area Severity Index; ESR: Erythrocyte Sedimentation Rate; RCP: Reactive C Protein; DMARDs: Disease modifying antirheumatic drugs . 
Table 2. Disease characteristics

N 278

\begin{tabular}{|c|c|}
\hline \multicolumn{2}{|l|}{ Inflammatory Bowel Disease } \\
\hline Crohn's disease, $n(\%)$ & $43(15)$ \\
\hline HBI & $2 \pm 3$ \\
\hline Ulcerative Colitis, $n(\%)$ & $93(32)$ \\
\hline MS & $2 \pm 2$ \\
\hline \multicolumn{2}{|l|}{ Rheumatological Disorders } \\
\hline Axial Arthritis & $9(3)$ \\
\hline ASDAS & $1.5 \pm .9$ \\
\hline Peripheral Arthritis & $60(20)$ \\
\hline DAS-28 & $2.2 \pm 1$ \\
\hline Systemic Sclerosis & $14(5)$ \\
\hline MSI & $3 \pm 3$ \\
\hline Systemic Lupus Erythematosus & $10(3)$ \\
\hline SELENA-SLEDAI & $3.5 \pm 5$ \\
\hline Vasculitis & $7(2)$ \\
\hline BVAS irmingham Vasculitis Activity Score & $1.2 \pm 2.2$ \\
\hline
\end{tabular}

Dermatological Disorders

Psoriasis

PASI

$1.7 \pm 1.8$

Atopic dermatitis

EASI

$27 \pm 1$

Disease duration (years)

$10 \pm 8$

$\operatorname{ESR}(\mathrm{mm} / \mathrm{h})$

$14 \pm 14$

$\mathrm{RCP}(\mathrm{mg} / \mathrm{l})$

$6 \pm 11$

Active Disease n (\%)

80 (29)

Surgery, $n(\%)$

17(6)

Medications, $n(\%)$

DMARDs

FANS

7 (2)

Mesalamine

Steroids

Biological

$95(23)$

Continuous variables are shown as mean \pm standard deviation, categorical variables are presented as number and proportion. Abbreviations: HBI: Harvey Bradshaw Index; MS: Mayo Score; ASDAS: Ankylosing Spondylitis Disease Activity Score; DAS-28: Disease Activity Score -28 ;MSI: Medsger Severity Index; SELENA-SLEDAI: Selena-Systemic Lupus Erythematosus Disease Activity Index; BVAS: Birmingham Vasculitis Activity Score; PASI: Psoriasis Activity Score Index; EASI: Eczema Area Severity Index; ESR: Erythrocyte Sedimentation Rate; RCP: Reactive C Protein; DMARDs: Disease modifying antirheumatic drugs . 


\section{Table 3 (on next page)}

Table 3. Model comparison between the unconstrained (2PL) and constrained (1PL) Graded Response Models

Abbreviations: BIC: Bayesian Information Criterion. logLik: Log Likelihood. LRT: Likelihood Ratio Test. DF: degrees of freedom. 
Table 3. Model comparison between the unconstrained (2PL) and constrained (1PL) Graded Response Models

\begin{tabular}{|c|c|c|c|c|c|}
\hline Model & BIC & logLik & $\Delta \chi^{2}$ & df & p value \\
\hline "Constrained (1PL) & "5080 & 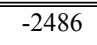 & & & \\
\hline Unconstrained (2PL) & 5045 & -2459 & 54 & 3 & $<.001$ \\
\hline
\end{tabular}

\section{1}




\section{Table 4 (on next page)}

Table 4. T-scores stratified for study group.

Abbreviations: CTR: Control group, IBD: Inflammatory Bowel Disease, RHEUMA:

Rheumatological disorder group, DERMA: Dermatological disorder group 
Table 4. Descriptive statistics of the T-scores stratified by study group

\begin{tabular}{|c|c|c|c|c|c|c|c|c|c|}
\hline Group & $\mathbf{n}$ & mean & sd & median & $\min$ & $\max$ & range & skew & kurtosis \\
\hline CTR & 280 & 48.33 & 8.22 & 47.2 & 37.3 & 76.1 & 38.8 & 0.34 & -0.36 \\
\hline IBD & 144 & 54.21 & 11.97 & 53.35 & 37.3 & 79.9 & 42.6 & 0.20 & -0.86 \\
\hline RHE & 100 & 55.77 & 9.92 & 57.1 & 37.3 & 79.9 & 42.6 & -0.23 & -0.52 \\
\hline DER & 43 & 54.93 & 12.54 & 57.1 & 37.3 & 79.9 & 42.6 & -0.03 & -1.08 \\
\hline
\end{tabular}

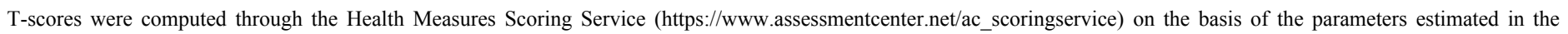

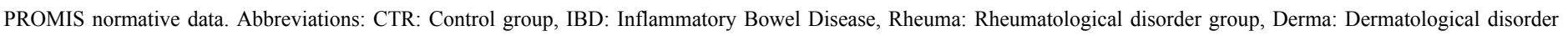
group 
Figure 1

Response frequency distribution.

Response frequency distribution across items of the custom 4-items Italian adaptation from the PROMIS Anxiety 8a short form. Panels A-D show items 1- 4 of the custom 4-items anxiety 165 short from the PROMIS anxiety 8 a short 
A
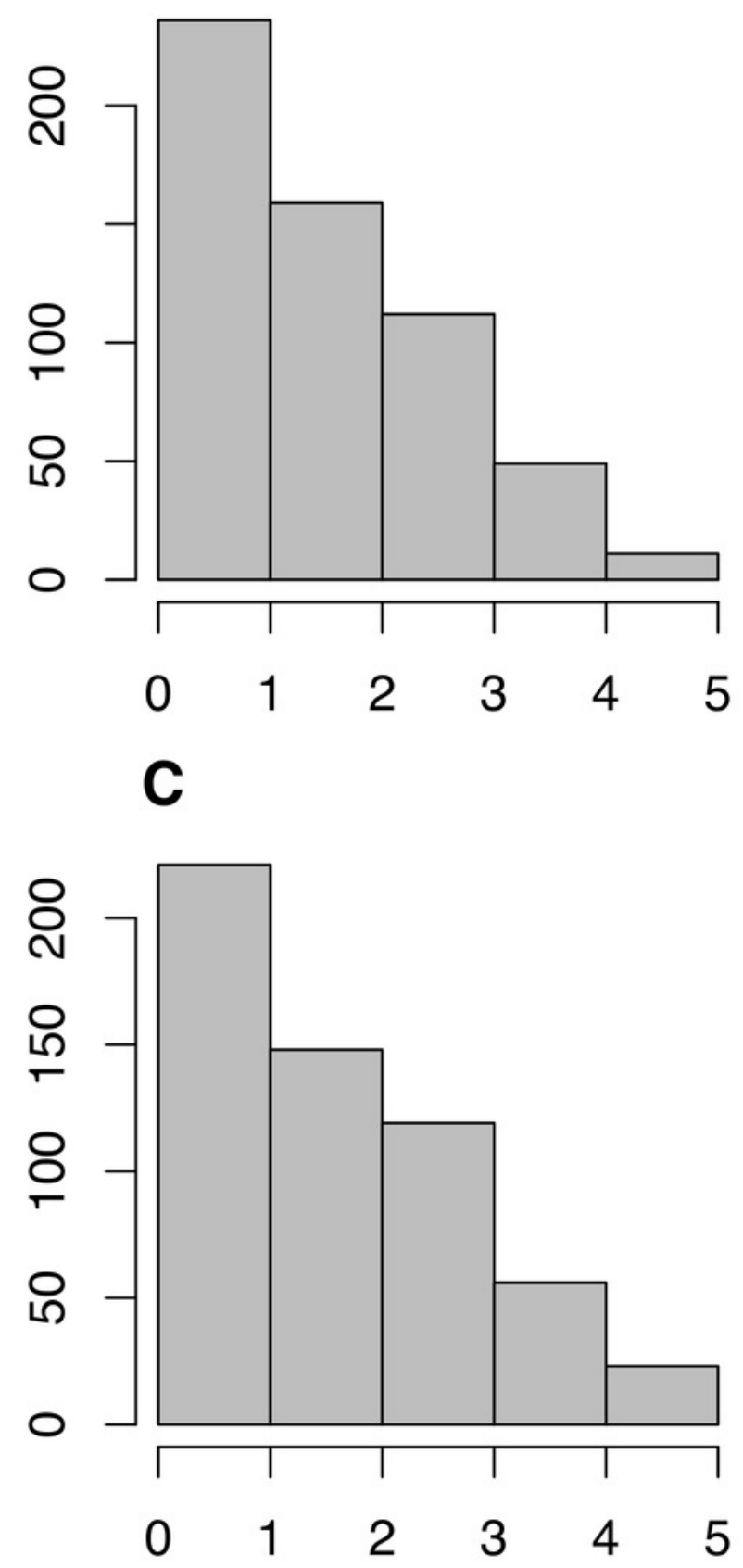

B
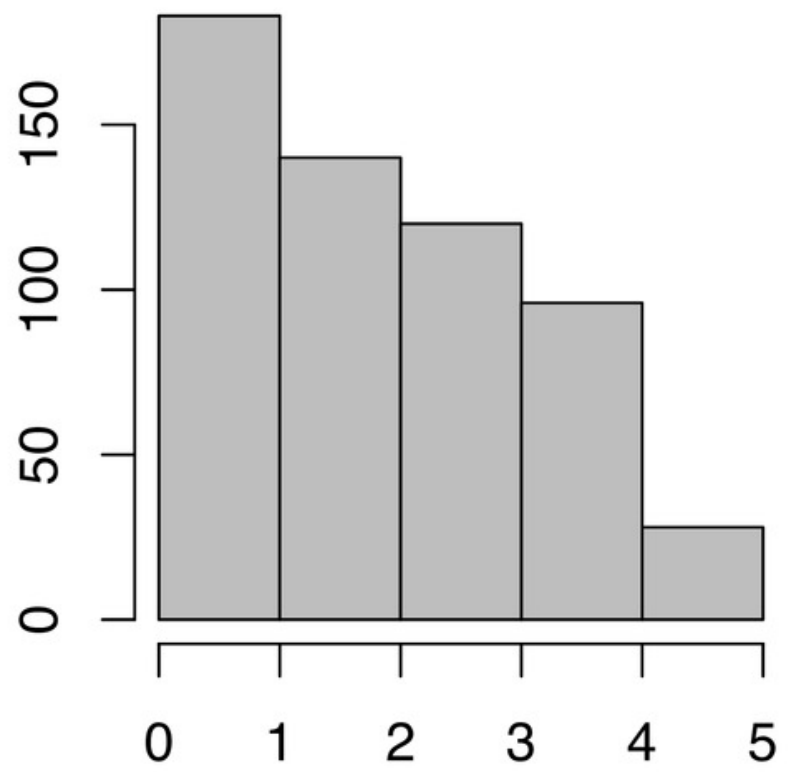

D

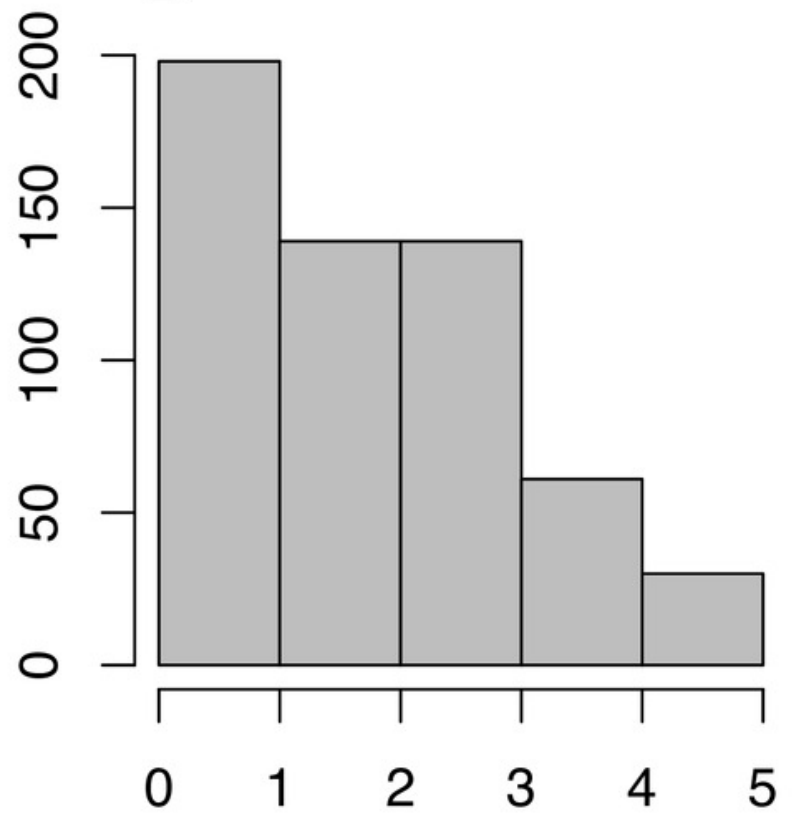


Figure 2

The scree plot plots the $i$-th factor against its eigenvalue

\section{Scree Plot}

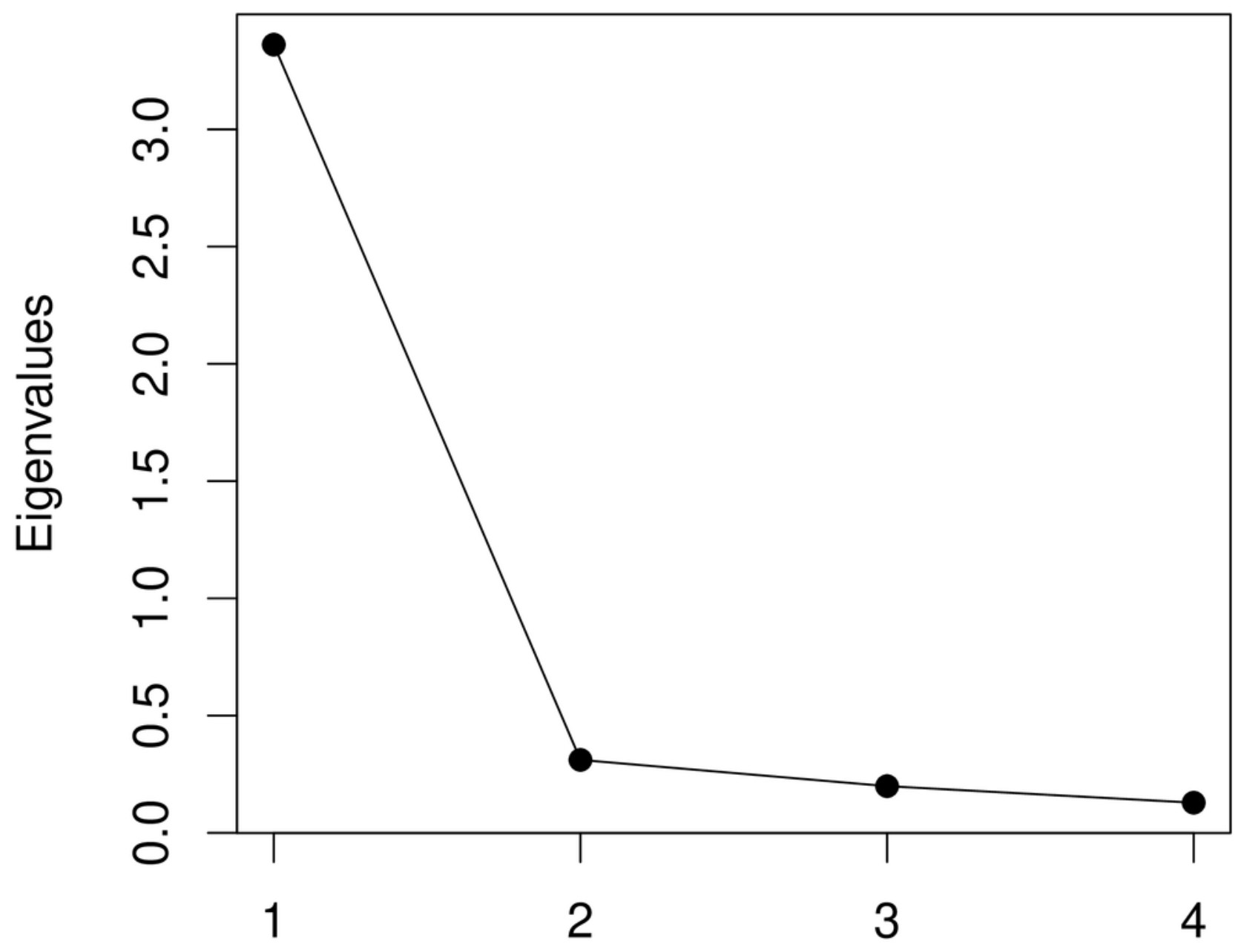

Factors 


\section{Figure 3}

Item Response Category Characteristic Curves of the custom 4-items Italian adaptation from the PROMIS Anxiety 8a short form.

Each line represents one response category on the 5-points Likert-type response. The y-axis represents the probability of choosing that response category. The $x$-axis represents different levels of the latent trait (Theta). The name of the items refers to the original enumeration in the 8a short form. 


\section{Item trace lines}

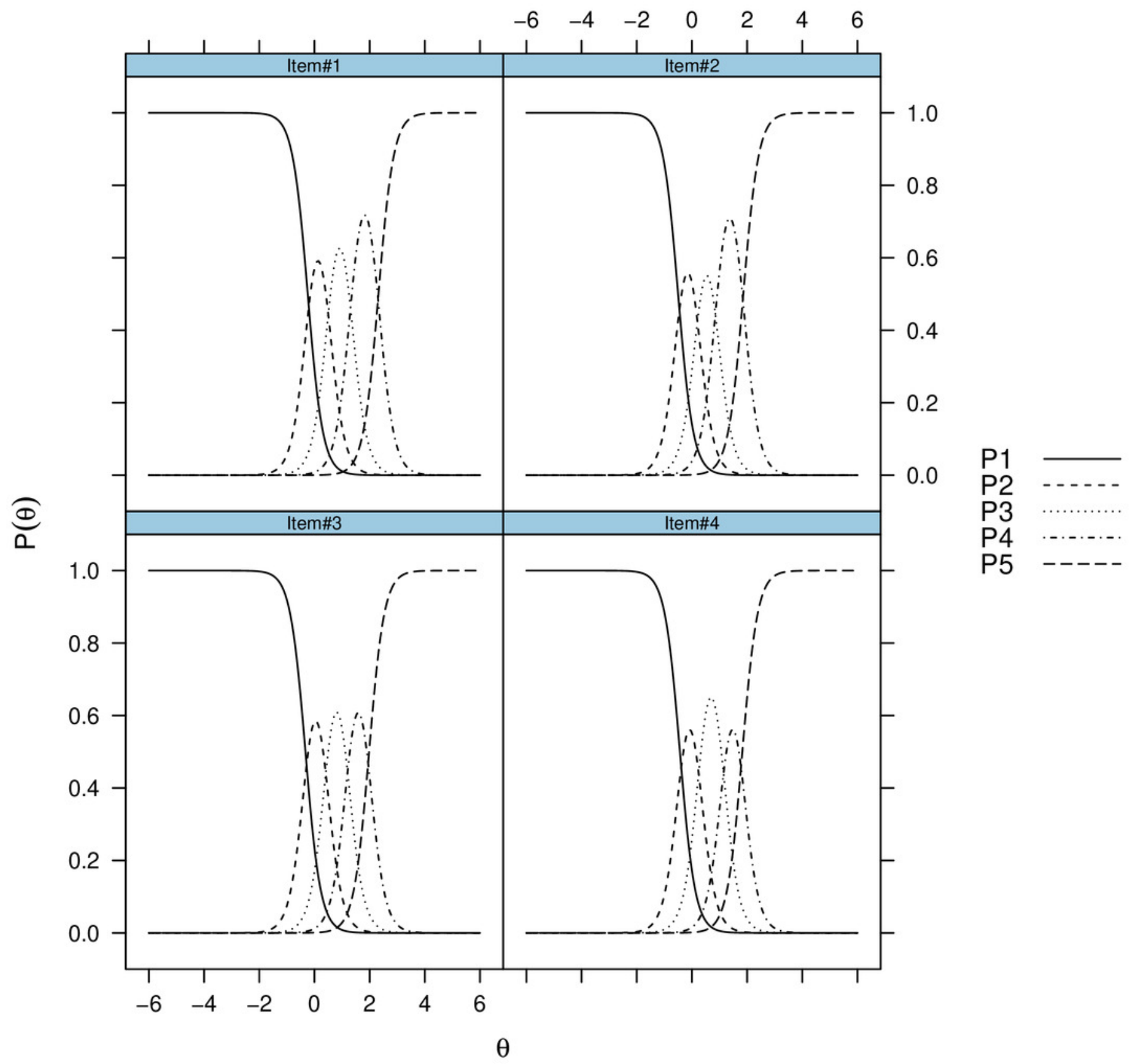


Figure 4

Test reliability plot, computed from the test information plot.

Levels of reliability (y-axis) as a function of the estimated latent trait level (Theta).

\section{Reliability}

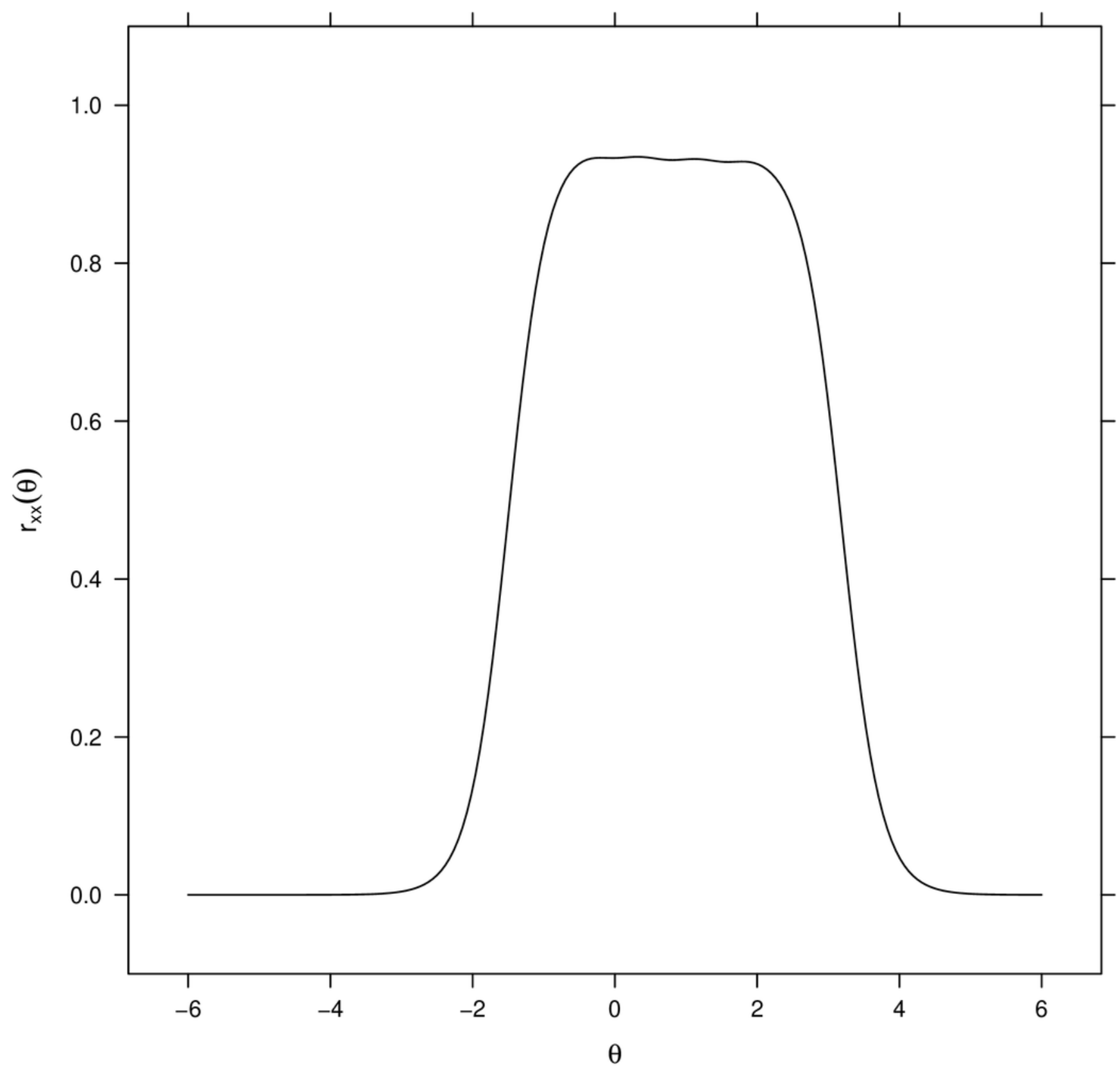


Figure 5

Least Square Means and 95\% confidence intervals of the T-scores of each group.

Abbreviations: $\mathrm{CTR}=$ Control group, $\mathrm{DER}=$ Dermatological disorder group, $\mathrm{IBD}=$ Inflammatory Bowel Disease group RHE = Rheumatological disorder group . 


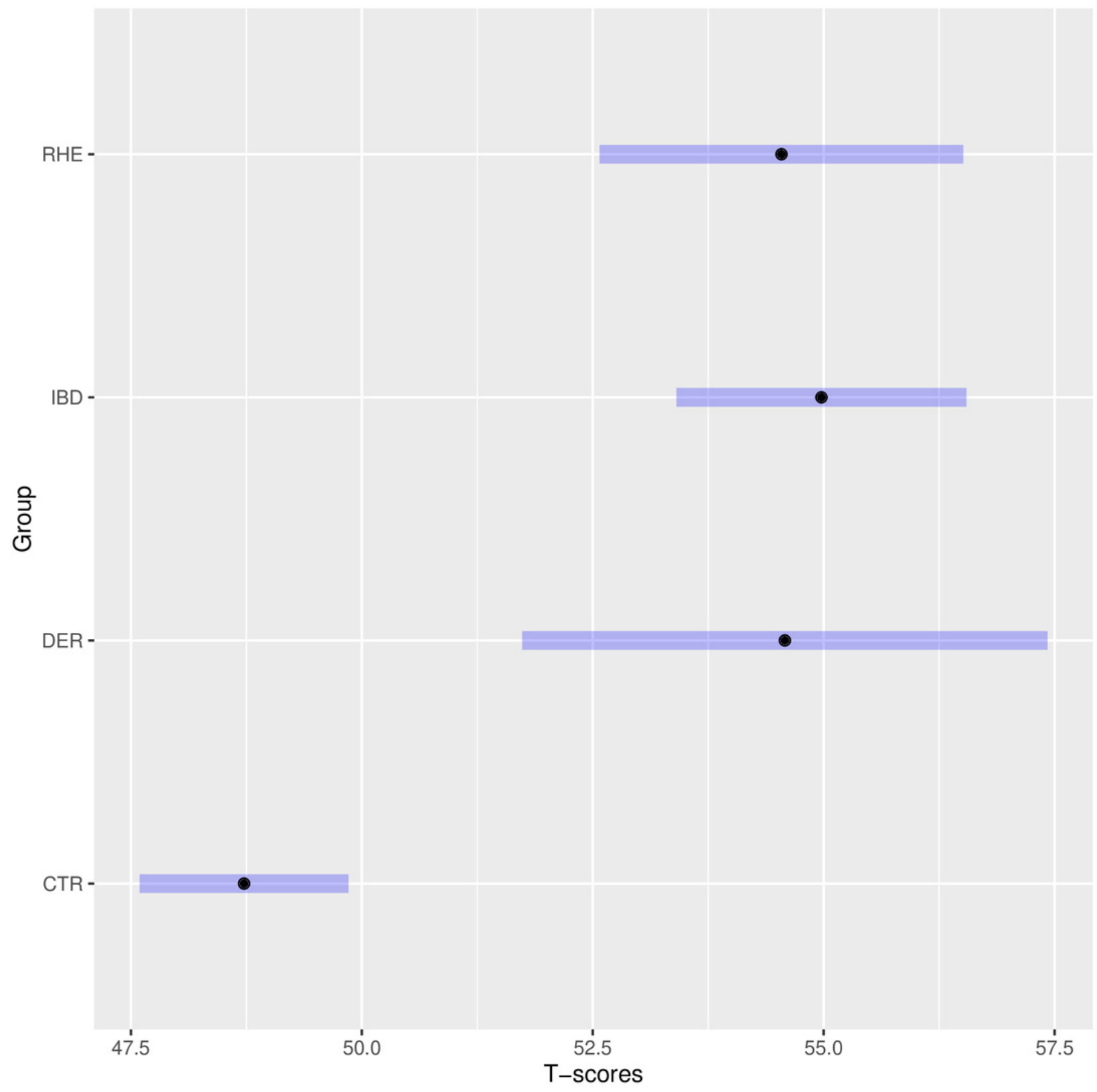

